\title{
Calibrated Automated Thrombin Generation in Frozen-Thawed Platelet-Rich Plasma to Detect Hypercoagulability
}

\author{
Véronique Regnault ${ }^{a}$ Suzette Beguin ${ }^{c}$ Thomas Lecompte ${ }^{a, b}$ \\ aINSERM ERIT-M 0323 et EA 3452, Faculté de Médecine, Université Henri Poincaré, et b Hématologie Biologique, \\ CHU de Nancy, Nancy, France; 'Synapse BV, Cardiovascular Research Institute, University of Maastricht, \\ Maastricht, The Netherlands
}

\section{Key Words}

Activated protein C $\cdot$ Factor VIII · Platelets . Thrombin generation

\begin{abstract}
To enhance the practical applicability of the calibrated automated thrombogram (CAT) we investigated whether frozen-thawed platelet-rich plasma (ft-PRP) can be used to assess the function of the protein $C$ inhibitory pathway, while preserving the natural phospholipid composition. Recalcified ft-PRP triggered with $0.5 \mathrm{pM}$ recombinant human tissue factor shows a median thrombin potential of $1,779 \mathrm{n} M \cdot \min$, against $1,576 \mathrm{n} M \cdot \min$ for fresh PRP. To obtain $\sim 70 \%$ inhibition, $6.7 \mathrm{nM}$ activated protein $C$ (APC) has to be added, instead of $25 \mathrm{nM}$ in fresh PRP; so the relative APC resistance of PRP appears to depend upon the presence of intact platelets. Factor VIII, added to normal ft-PRP to obtain a concentration of $3.3 \mathrm{U} / \mathrm{ml}$, increases the thrombin potential in the presence of APC 1.5-fold, from 524 to $808 \mathrm{nM} \cdot \mathrm{min}$, in keeping with previously published increases in thrombotic risk in patients with high factor VIII levels. We conclude that thrombography in ft-PRP, with and without added APC, can be used to assess known risk factors for thrombosis, which allows the design of large clinical studies aimed at proving the relationship between thrombin potential and
\end{abstract} clinical outcome.

Copyright $@ 2003$ S. Karger AG, Basel

\section{Introduction}

Assessing the individual thrombotic risk by analysis of genetic polymorphisms and coagulation factors still leads to considerable discrepancies between the general risk associated with these abnormalities and the risk experienced by the individual patient. Measuring the effect of the combined genetic, acquired and environmental factors on the function of the hemostatic system ("phenotyping') is a more promising approach [1]. Thrombin activity measured in clotting plasma is a promising tool for such phenotyping [2]. We introduced the calibrated automated thrombogram (CAT) in our clinical laboratory in order to investigate the suitability of this technique for clinical diagnosis and treatment. As a first result, we demonstrated in platelet-rich plasma (PRP), stimulated with a low concentration $(0.5 \mathrm{p} M)$ of recombinant human tissue factor (rh-TF), acquired resistance to activated protein $\mathrm{C}$ (APC) in patients with lupus anticoagulants despite a prolongation of the lag time of the thrombin burst [3].

Given the essential role of platelets in blood coagulation [4, 5], it is of paramount importance to include platelets in the system tested. To avoid artifacts due to accidental platelet activation, this requires the use of carefully prepared PRP, tested within 90 min after venipuncture. This, however, is not easily accomplished in large multicenter studies that are required to ascertain correlations between clinical and laboratory data. We therefore inves-

Véronique Regnault

INSERM ERIT-M 0323 'Nouvelles approches anti-thrombotiques' EA 3452

Faculté de Médecine, Université Henri Poincaré, Boite Postale 184

FR-54505 Vandoeuvre-lès-Nancy (France)

Tel. +333836834 70, Fax +333833834 79, E-mail regnault@hemato.u-nancy.fr 
tigated in how far frozen-thawed PRP (ft-PRP) would be a convenient alternative to fresh PRP. To track down APC resistance, our tests included the effects of adding APC and thrombomodulin (TM) and we also investigated the effect of addition of factor VIII, a well established and common risk factor for thrombosis, per se and via decreased sensitivity to APC [6].

\section{Materials and Methods}

\section{Proteins and Reagents}

Bovine serum albumin (BSA) and all chemicals were from Sigma (St. Louis, Mo., USA). rh-TF, relipidated and not containing polybrene or calcium, was a kind gift from Dade Behring (Marburg, Germany). The fluorogenic substrate Z-Gly-Gly-Arg-AMC was from Bachem (Bubendorf, Switzerland). Human factor VIII was provided by LFB (Lille, France). The calibrator with a constant, known, thrombin-like activity was from Synapse BV (Maastricht, The Netherlands). Soluble rh-TM (rhs-TM) composed of all the extracellular domains of TM without chondroitin sulfate chains [7] was a kind gift of Asahi (Japan). Human APC was prepared in-house as described previously [8]. For the clot-based assay [9], we used the STA coagulometer from Diagnostica Stago (Asnières, France) with the automated APTT reagent from Organon Teknika (Akzo-Nobel, The Netherlands) as a trigger.

\section{Blood and Plasma}

Blood from healthy volunteers was obtained by venipuncture with minimum trauma via a 21-gauge needle and collected into $0.129 \mathrm{~mol} / \mathrm{l}$ sodium citrate $(9: 1 \mathrm{vol} / \mathrm{vol})$. PRP was prepared by centrifugation at $194 \mathrm{~g}$ for $10 \mathrm{~min}$ at room temperature. Platelet counts were measured using a Micros $60 \mathrm{ABX}$ model (Montpellier, France) and adjusted to $150 \times 10^{9}$ platelets/ 1 in PRP by addition of autologous platelet-poor plasma (PPP), which was obtained by double centrifugation at 2,500 $\mathrm{g}$ for $10 \mathrm{~min}$. Exclusion criteria were factor $\mathrm{V}$ 506Q polymorphism or medical treatment. All individuals exhibited normal values for routine clotting tests, clotting factor levels and platelet counts. Factor VIII levels were measured with a one-stage clotting assay using a commercially available plasma deficient in factor VIII. PRP was used within $90 \mathrm{~min}$ after venipuncture, and an aliquot was frozen at $-80^{\circ} \mathrm{C}$. Frozen PRP was thawed at $37^{\circ} \mathrm{C}$ for $15 \mathrm{~min}$ and tested within $30 \mathrm{~min}$ thereafter.

\section{Fluorogenic Measurement of Thrombin Activity}

The thrombograms were recorded at $37^{\circ} \mathrm{C}$ according to Hemker et al. $[10,11]$ in a microtiter plate fluorometer (Fluoroskan Ascent, ThermoLabsystems, Helsinki, Finland) equipped with a 390/460 filter set for excitation and emission and using the dedicated software program (Thrombinoscope, Synapse BV). Round-bottom 96-well Greiner blue plates (reference No. 650204) were used. Coagulation was triggered by recalcification in the presence of $0.5 \mathrm{p} M$ rh-TF. In each well, $5 \mu$ l of rh-TF solution in $20 \mathrm{~m} M$ Hepes, $140 \mathrm{~m} M \mathrm{NaCl}, \mathrm{pH}$ 7.35 , containing $5 \mathrm{~g} / \mathrm{l} \mathrm{BSA}$ (HBS buffer) and $15 \mu \mathrm{l}$ of HBS buffer or APC or rhs-TM in HBS buffer were added to $80 \mu \mathrm{l}$ of PRP. Experiments were carried out in triplicate. A calibrator well in which $20 \mu \mathrm{l}$ of calibrator were added to $80 \mu$ of PRP was run in parallel for each plasma. The plate was placed in the fluorometer for $5 \mathrm{~min}$. Then

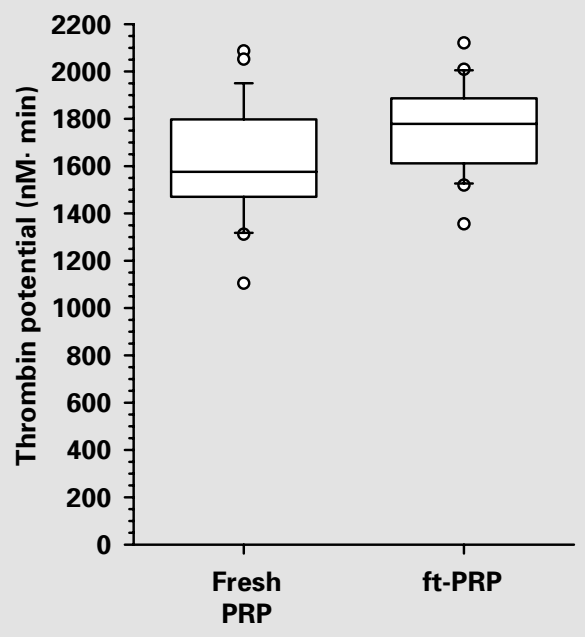

Fig. 1. Comparison of thrombin potential values obtained with fresh PRP and ft-PRP. The generation of thrombin through the TF-initiated extrinsic coagulation pathway was monitored using the calibrated thrombogram as detailed in the Methods. Coagulation was triggered with $0.5 \mathrm{p} M$ rh-TF in the presence of $16.7 \mathrm{mMCaCl}_{2}$. PRP was used within $90 \mathrm{~min}$ after venipuncture. The thrombin potential was assessed as the area under the curve. The box indicates the 25 th and 75 th percentile, the line inside the box the median, error bars the 10 th and 90th percentiles.

$20 \mu \mathrm{l}$ of a mixture of $2.5 \mathrm{~m} M$ fluorogenic substrate in Hepes $20 \mathrm{~m} M$, $\mathrm{pH} 7.35$, containing $60 \mathrm{~g} / \mathrm{BSA}$ and $100 \mathrm{~m} M \mathrm{CaCl}_{2}$ was dispensed in each well by the instrument, and the fluorescent signal was recorded with a measurement interval of $15 \mathrm{~s}$. The molar amount of thrombin present in clotting plasma was calculated from the signal of wells in which thrombin is generated and the calibration signal as previously described [12]. The total amount of thrombin activity (i.e. the thrombin potential) was assessed as the area under the curve.

\section{Statistical Analysis}

The comparison between thrombin potentials obtained with 'fresh' PRP and ft-PRP was assessed by means of the two-sided Wilcoxon test for pairs. The correlation between the factor VIII level and the thrombin potential was analyzed by the Spearman rank correlation coefficient. 

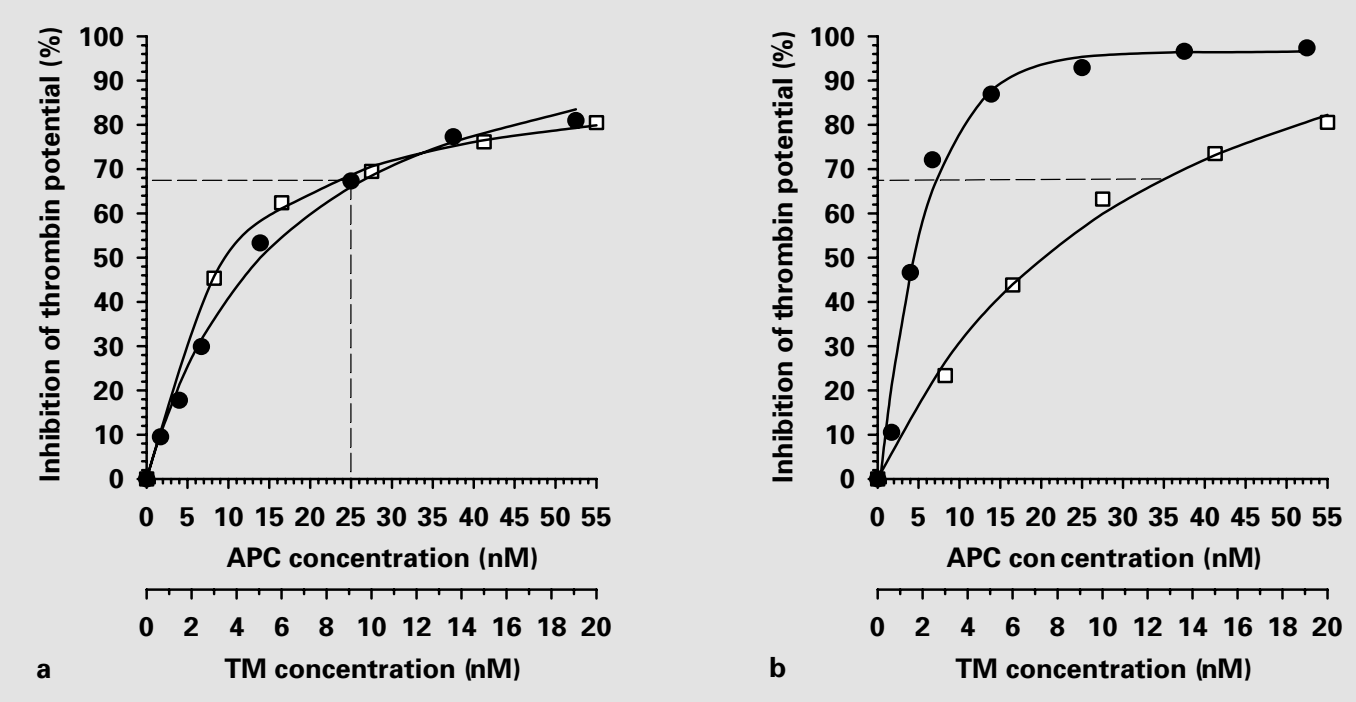

Fig. 2. Effect of TM and APC on thrombin potential. a Fresh PRP. b ft-PRP. Inhibition of thrombin activity was assessed in the presence of TM $(\square)$ and APC (๑). Dashed horizontal line: 70\% inhibition; dashed vertical line: concentrations of TM and APC at which this inhibition is obtained.

\section{Results}

\section{Thrombin Potential in Fresh PRP and ft-PRP}

As expected, the burst of thrombin generation started earlier, and maximal thrombin activity (the thrombin peak) was higher with ft-PRP than with fresh PRP. There was a slight but significant increase $(\sim 10 \%)$ in the endogenous thrombin potential with ft-PRP as compared with fresh PRP (fig. 1). In our population of 20 normal controls, the median values of thrombin potential were 1,576 and $1,779 \mathrm{n} M \cdot \min$ for PRP and ft-PRP, respectively $(\mathrm{p}<$ $0.005)$.

\section{Inhibition of Thrombin Potential by APC}

In PRP preparations from healthy volunteers, the addition of APC led to a concentration-dependent decrease in thrombin potential. Similar inhibition patterns were observed with added, exogenous APC and endogenous APC, formed during thrombin generation via rhs-TM (fig. 2a) added to PRP. A 70\% decrease in thrombin potential was observed with $25 \mathrm{n} M$ APC or with $10 \mathrm{n} M$ rhs-TM. In ft-PRP, this inhibition is obtained with $\sim 6.7 \mathrm{n} M$ exogenous APC or $\sim 12 \mathrm{n} M$ rhs-TM (fig. 2). A batch-to-batch comparison of three in-house APC preparations showed superimposable thrombograms both with

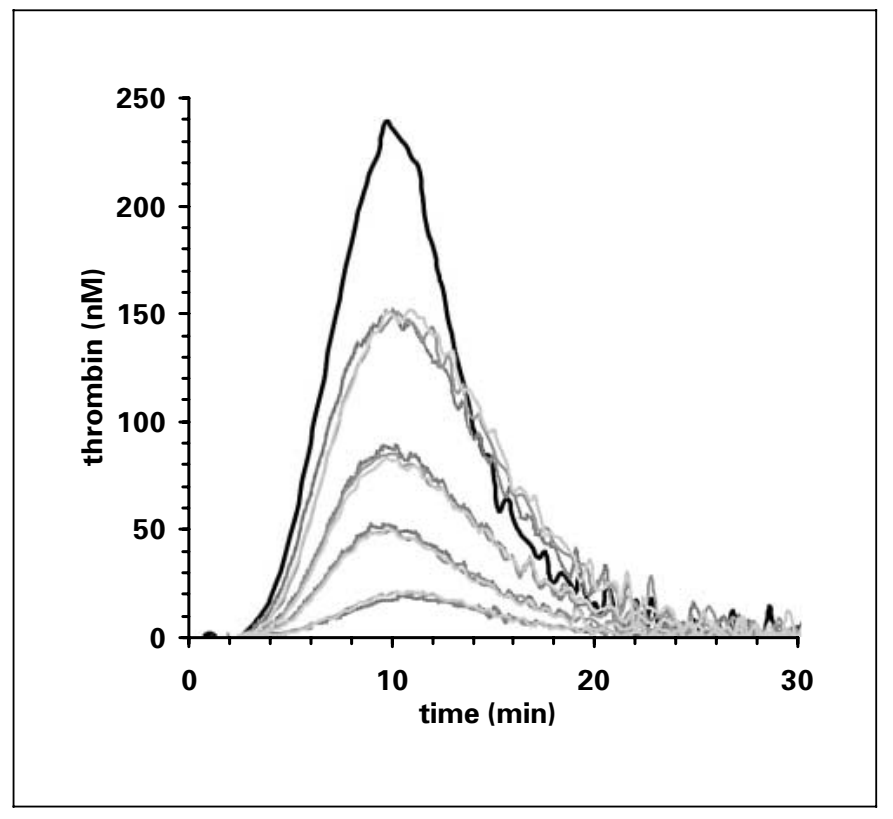

Fig. 3. Batch-to-batch reproducibility of the APC anticoagulant function on the thrombin course (thrombograms). The anticoagulant effect of three batches of APC was assessed with ft-PRP. APC concentrations from top to bottom $0,1.7,4,6.7$ and $13.9 \mathrm{n} M$.

Pathophysiol Haemost Thromb 2003;33:23-29 
Fig. 4. Intra-individual variation in thrombin potential. The thrombin potential was measured 8 times over a 17 -month period. The box indicates the 25 th and 75 th percentile, the line inside the box the median; error bars the 10th and 90th percentiles.
Fig. 5. APC sensitivity ratio (clot-based assay) as a function of factor VIII level. The original APC sensitivity clot-based assay was performed with PPP from 123 patients with a history of thrombosis and referred to the specialized clinical laboratory of our hospital for detection of thrombophilia. Regression line, $95 \%$ confidence interval (dotted line) and $95 \%$ prediction interval (dashed line).
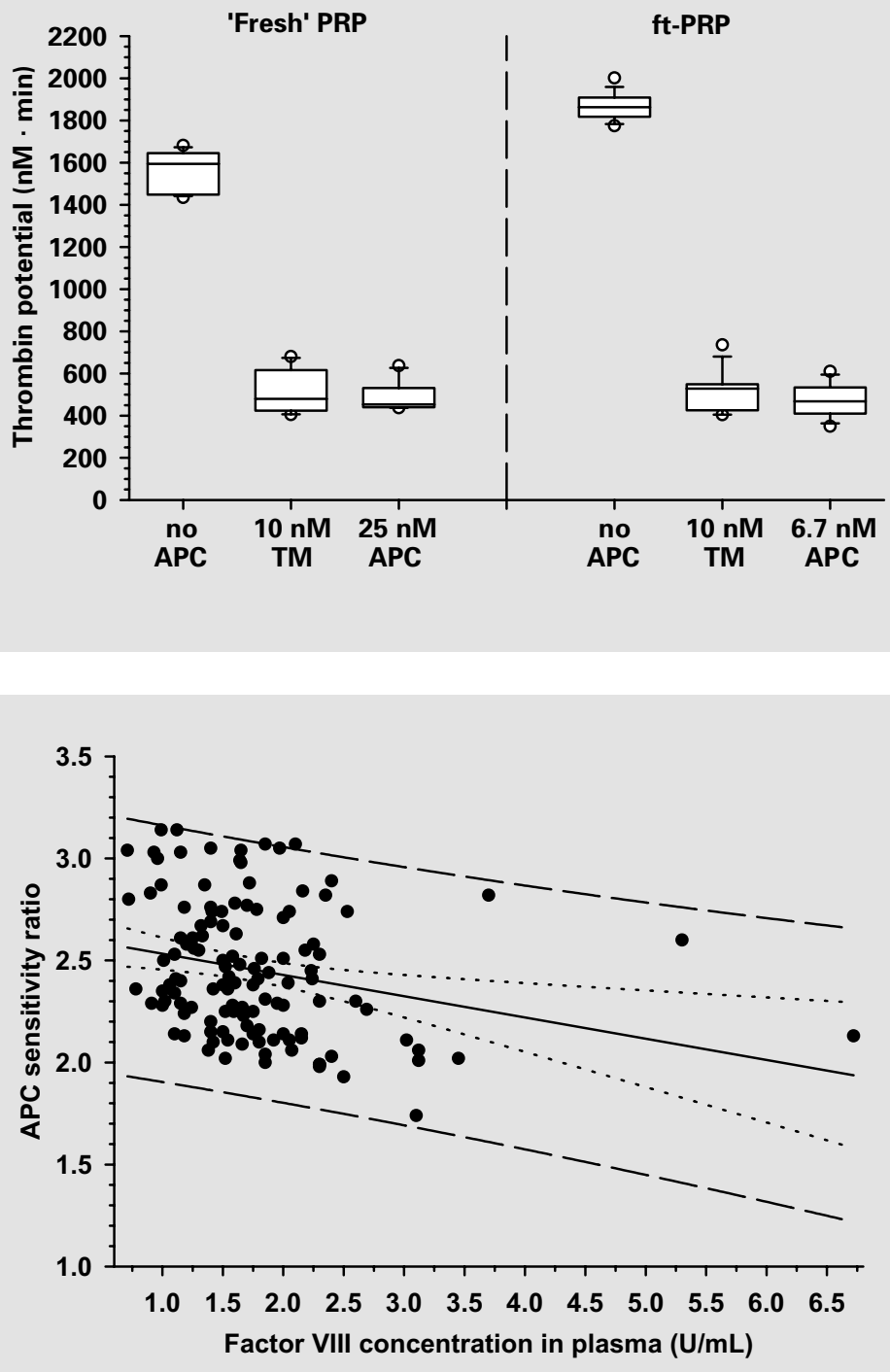

fresh PRP and ft-PRP (fig. 3). Similarly, low intra-individual variations were observed with fresh PRP and ftPRP (fig. 4).

\section{Effect of Addition of Purified Factor VIII to Normal \\ Plasma}

Our data confirmed an association between factor VIII level and sensitivity to APC, as assessed with the original, clot-based assay and our in-house APC preparation, in patients referred to the specialized clinical unit for the detection of thrombophilia (fig. 5). We then measured the effect on thrombin generation in normal PRP of added purified human factor VIII at levels encountered in thrombosis-prone patients ( 95 percentile). As factor VIII increased, the thrombin potential increased, and the sensitivity to APC decreased (fig. 6), both in fresh PRP and in ft-PRP ( $p=0.045$ for each of the 4 experimental conditions). Factor VIII, added to normal ft-PRP to obtain a concentration of $3.3 \mathrm{U} / \mathrm{ml}$, increases the thrombin potential in the presence of APC 1.5-fold, from 524 to 808 $\mathrm{n} M \cdot \min$. 


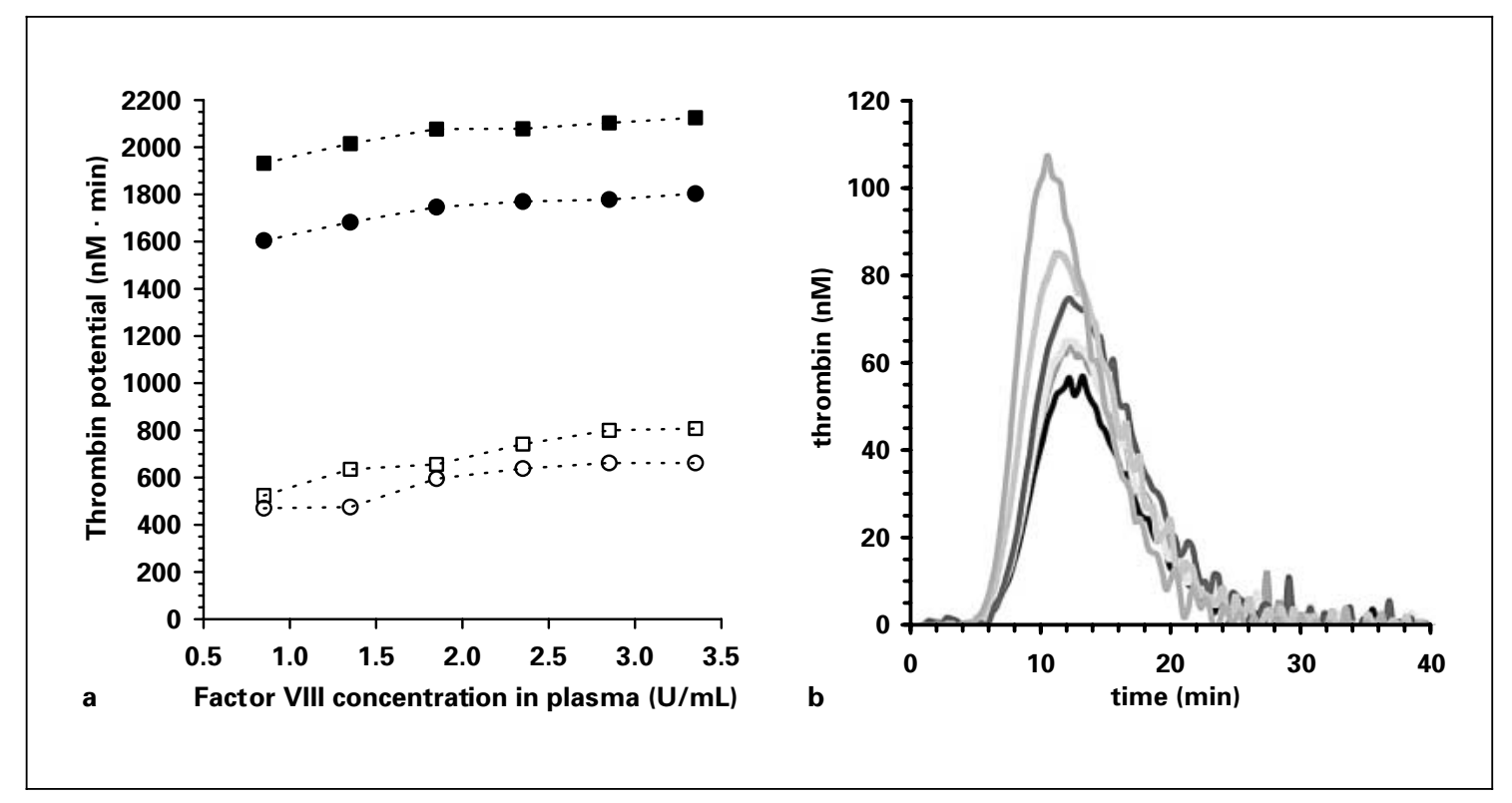

Fig. 6. Dependence of the thrombin potential on FVIII concentration in the absence or presence of an APC system. a Experiments were performed with fresh PRP $(\bullet)$ and ft-PRP $(\square)$ in the absence or presence of 25 and $6.7 \mathrm{n} M$ APC for PRP $(O)$ and ft-PRP $(\square)$, respectively. Results are the mean of three tests with iterative blood samples of the same normal subject. b Typical thrombograms with ft-PRP in the presence of $6.7 \mathrm{n} M$ APC. Factor VIII concentrations from bottom to top $0.8,1.3,1.8,2.3,2.8$ and $3.3 \mathrm{U} / \mathrm{ml}$.

\section{Discussion}

In a previous study, we have shown that for measuring the effect of lupus anticoagulants the sensitivity of the test is increased if the platelet phospholipids of the patient himself are used rather than exogenously added phospholipids [3]. Like any physiological function test, thrombin generation in fresh PRP requires the presence of the patient. For practical purposes it is more convenient to work on stored material; therefore, in the present study, we investigated whether ft-PRP can be used to assess the effect of the protein $\mathrm{C}$ system on thrombin generation.

The time course of thrombin differs between fresh PRP and ft-PRP, in that the thrombin burst is earlier as well as higher in ft-PRP, although the thrombin potential is not much higher (fig. 1). This is probably due to the fact that after freezing and thawing, with its concomitant membrane damage, the exposure of procoagulant phospholipids is no longer dependent upon the physiological membrane scrambling of the intact platelet [13]. Since TF pathway inhibitor (TFPI) is the key regulator of the initiation phase of thrombin generation [14], the observation that the thrombin potential is somewhat higher with $\mathrm{ft}$ PRP than with fresh PRP could be explained by a lesser effectiveness of TPFI when the bulk of activated factor X is rapidly produced.

As in PPP with added PL and in fresh PRP [11], ftPRP also showed low intra-individual (day-to-day) variations. The distribution of thrombin potential values confirms a relatively large interindividual variability.

For measuring APC sensitivity, either exogenous APC or endogenous PC, which is activated in the presence of added TM, can be used. In the latter case not only APC sensitivity of factors V and VIII is tested but also the APC forming system. APC might be preferred since it is a natural soluble protein, which is easily obtained in a reproducible manner (fig. 3). TM, in contrast, is a transmembrane protein that is currently used as a recombinant soluble form lacking the transmembrane domain and its physiological surroundings. Whatever the source of APC, this inhibitor needs a phospholipid surface for its activity, the optimal composition of the surface being different from the optimal composition for procoagulant reactions, so that using the patients' own platelet phospholipids gives additional information on the equilibrium of the two processes in vivo.

With respect to the concentration of APC used, a peak circulating APC level of $23 \mathrm{n} M$ was reported in baboons 
infused with thrombin [15]. Since the concentrations of protein $\mathrm{C}$ and prothrombin in the baboon are in the normal human range [16], we can speculate that a similar circulating level of APC is reached in human plasma at the site where thrombin is formed; we therefore chose $25 \mathrm{nM}$, which gave a convenient $70 \%$ inhibition of thrombin generation in normal controls. In ft-PRP we then sought the concentration that gave a similar inhibition.

Our data show an increased sensitivity to APC in ftPRP as compared with PRP. Taube et al. [17] reported progressive APC resistance with increasing platelet activation, including a freeze-thawing cycle. This is only an apparent discrepancy because Taube et al. [17] based their conclusion on the use of clotting times. Also, in our study, we found that the response to APC, expressed as a ratio of the lag times before the burst of thrombin (roughly equivalent to the clotting time) in the presence or absence of APC, was lower with ft-PRP than with PRP (data not shown). This again emphasizes the importance of measuring the whole thrombin generation curve rather than clotting times only.

Perhaps more intriguing is the similar, or even slightly decreased sensitivity of ft-PRP to endogenous APC formed during thrombin generation in the presence of added TM. It has been demonstrated that platelets release an inhibitor to APC during platelet activation [18] and a time-dependent formation of a complex between this inhibitor and APC has been reported [19]. Also, phosphatidylethanolamine has been shown to be essential for optimal APC activity [20]. It is highly probable that frozenthawed platelets, contrary to intact platelets, provide phosphatidylethanolamine right at the beginning of the reaction, thus enabling APC to engage in anticoagulant activity immediately. On the other hand, an inhibitor that is normally secreted in the neighborhood of the platelet surface is probably much diluted in the bulk of ft-PRP, and consequently the time-dependent neutralization of APC becomes less efficient.

Regarding the effect of factor VIII, it is well established that elevated levels of factor VIII are associated with a thrombotic risk [21, 22] and decreased APC sensitivity [6]. Here we report that the thrombin potential in the presence of APC increases about 50\% with increasing factor VIII levels. Of note, the clot-based assay seems to underestimate the impact of factor VIII, since the decrease in the APC sensitivity ratio is only $10 \%$ (from approximately 2.5 to 2.3 , for plasma levels of factor VIII between 1 and $3 \mathrm{U} / \mathrm{ml}$ ). Furthermore, using thrombography, the effect on the lag time ( clotting time) was of the same order of magnitude as that seen with the original clot-based assay (data not shown), and much less conspicuous than the effect on thrombin potential.

We conclude that with the CAT methodology thrombography can be conveniently implemented in clinical research. It appears that ft-PRP retains several features of fresh PRP, so that it may be used for the detection of APC resistance if it is impossible to work in the vicinity of the patient. We also noted that known risk factors for thrombosis, e.g. factor VIII (this study) or lupus anticoagulants [3], definitely affect this test, and thus it is apparently a promising and attractive candidate to assay the ensemble of genetic and other effects on the hemostatic system determining the individual thrombotic risk.

\section{Acknowledgments}

We thank the staff of the Hematology Laboratory of the CHU of Nancy for technical assistance, the Centre d'Investigation Clinique 9501, CHU of Nancy and INSERM, for blood sampling in selected healthy volunteers, and Dr. D. Wahl, PhD in clinical epidemiology, for statistical advice. This work was supported by INSERM and the 'Région Lorraine'.

\section{References}

1 Laffan M, Tuddenham E: Assessing thrombotic risk. BMJ 1998;317:520-523.

2 Hemker HC, Béguin S: Phenotyping the clotting system. Thromb Haemost 2000;84:747751.

3 Regnault V, Béguin S, Wahl D, De Maistre E, Hemker HC, Lecompte T: Thrombinography shows acquired resistance to activated protein $\mathrm{C}$ in patients with lupus anticoagulants. Thromb Haemost 2003;89:208-212.

4 Heemskerk JWM, Bevers EM, Lindhout T: Platelet activation and blood coagulation. Thromb Haemost 2002;88:186-193.
5 Monroe DM, Hoffman M, Roberts HR: Platelets and thrombin generation. Arterioscler Thromb Vasc Biol 2002;22:1381-1389.

6 Soria JM, Almasy L, Souto JC, Buil A, Martinez-Sanchez E, Mateo J, Borell M, Stone WH, Lathrop M, Fontcuberta J, Blangero J: A new locus on chromosome 18 that influences normal variation in activated protein $\mathrm{C}$ resistance phenotype and factor VIII activity and its relation to thrombosis susceptibility. Blood 2003; 101:163-167.
7 Gomi K, Zushi M, Honda G, Kawahara S, Matsuzaki O, Kanabayashi T, Yamamoto I, Mauryama I, Suzuki K: Antithrombotic effect of recombinant human thrombomodulin on thrombin-induced thromboembolism in mice. Blood 1990; 75:1396-1399.

8 Regnault V, De Maistre E, Geschier C, Briquel ME, André E, Stoltz JF, Lecompte T: A new fast one-step immunopreparation for activated protein C. Thromb Haemost 1995;73:1365. 
9 Dahlbäck B, Carlsson M, Svensson PJ: Familial thrombophilia due to a previously unrecognized mechanism characterized by poor anticoagulant response to activated protein C: Prediction of a cofactor to activated protein C. Proc Natl Acad Sci USA 1993;90:1004-1008.

10 Hemker HC, Giesen PLA, Ramjee M, Wagenvoord R, Béguin S: The thrombogram: monitoring thrombin generation in platelet rich plasma. Thromb Haemost 2000;83:589-591.

11 Hemker HC, Giesen P, Al Dieri R, Regnault V, de Smedt E, Wagenvoord R, Lecompte T, Béguin $\mathrm{S}$ : Calibrated automated thrombin generation measurement in clotting plasma. Pathophysiol Haemost Thromb 2003;33:4-15.

12 Hemker HC, Giesen P, Al Dieri R, Regnault V de Smedt E, Wagenvoord R, Lecompte T, Béguin $\mathrm{S}$ : Calibrated automated thrombin generation measurement in clotting plasma. Pathophysiol Haemost Thromb 2003;33:4-15.
13 Rosing J, van Rijn JLMM, Bevers EM, van Dieijen G, Comfurius P, Zwaal RF: The role of activated human platelets in prothombin and factor X activation. Blood 1985;65:319-332.

14 Mann KG, Butenas S, Brummel K: The dynamics of thrombin formation. Arterioscler Thromb Vasc Biol 2003;23:17-25.

15 Taylor FB, Peer GT, Lockhart MS, Ferrell G, Esmon CT: Endothelial cell protein $\mathrm{C}$ receptor plays an important role in protein $\mathrm{C}$ activation in vivo. Blood 2001;97:1685-1688.

16 Hampton JW, Matthew C: Similarities between baboon and human blood clotting. J Appl Physiol 1966;21:1713-1716.

17 Taube J, McWilliam N, Luddington R, Byrne $\mathrm{C}$, Baglin $\mathrm{T}$ : Activated protein $\mathrm{C}$ resistance: Effect of platelet activation, platelet-derived microparticles, and atherogenic lipoproteins. Blood 1999;93:3792-3797.

18 Jane SM, Mitchell CA, Hau L, Salem HH: Inhibition of activated protein $\mathrm{C}$ in platelets. $\mathrm{J}$ Clin Invest 1989;83:222-226.
19 Nishioka J, Ning M, Hayashi T, Suzuki K: Protein $\mathrm{C}$ inhibitor secreted from activated platelets efficiently inhibits activated protein $\mathrm{C}$ on phosphatidylethanolamine of platelet membrane and microvesicles. J Biol Chem 1998; 273:11281-11287.

20 Smirnov MD, Esmon CT: Phosphatidylethanolamine incroporation into vesicles selectively enhances factor $\mathrm{Va}$ inactivation by activated protein C. J Biol Chem 1994;269:816-819.

21 Koster T, Blann AD, Briet E, Vandenbroucke JP, Rosendaal FR: Role of clotting factor VIII in effect of von Willebrand factor on occurrence of deep-vein thrombosis. Lancet 1995; 345:152-155.

22 Kamphuisen PW, Eikenboom JCJ, Bertina RM: Elevated factor VIII levels and the risk of thrombosis. Arterioscler Thromb Vasc Biol 2001;21:731-738. 